\title{
Association of Small Dense Low-Density Lipoprotein Cholesterol with Stroke Risk, Severity and Prognosis
}

Peiyang Zhou ${ }^{1}$, Jincheng Liu ${ }^{1}$, Lingyun Wang ${ }^{1}$, Wenmin Feng ${ }^{1}$, Zhihua Cao ${ }^{1}$, Pu Wang ${ }^{1}$, Guangzhi Liu ${ }^{1}$, Chenglin Sun ${ }^{1}$, Yan Shen ${ }^{2}$, Lijun Wang ${ }^{3,4}$, Jiahuan $\mathrm{Xu}^{3,4}$, Peng Meng ${ }^{3,4}$, Ziwei $\mathrm{Li}^{3,4}$, Wang-yang $\mathrm{Xu}^{3,4}$ and Xifa Lan ${ }^{5}$

Peiyang Zhou and Jincheng Liu contributed equally to this work.

Wang-yang $\mathrm{Xu}$ and Xifa Lan are joint senior authors.

${ }^{1}$ Xiangyang No.1 People's Hospital, Hubei University of Medicine, Xiangyang, China.

${ }^{2}$ Ruijin Hospital, Affiliated to Shanghai Jiao Tong University School of Medicine, Shanghai, China

${ }^{3}$ Biotecan Medical Diagnostics Co., Ltd, Zhangjiang Center for Translational Medicine, Shanghai, China

${ }^{4}$ Shanghai Zhangiiang Institute of Medical Innovation, Shanghai 201204, China

${ }^{5}$ Qinhuangdao No.1 Hospital, Qinhuangdao, China

Aim: To investigate the association of small dense low-density lipoprotein cholesterol (sdLDL-C) and acute ischemic stroke (AIS) in terms of risk, severity, and outcomes. Prediction models were established to screen high-risk patients and predict prognosis of AIS patients.

Methods: We enrolled in this study 355 AIS patients and 171 non-AIS controls. AIS was subtyped according to TOAST criteria, and the severity and outcomes of AIS were measured. Blood glucose and lipid profiles including total cholesterol, triglyceride, and lipoproteins were measured in all patients using automatic measure. Lipoprotein subfractions were detected by the Lipoprint LDL system.

Results: As compared with the non-AIS control group, the AIS group had higher sdLDL-C levels. Pearson correlation analysis revealed that the sdLDL-C level and risk of AIS, especially non-cardioembolic stroke, were positively correlated. The area under the curve of sdLDL-C for AIS risk was $\mathbf{0 . 6 6 5}$, better than that of other lipids. Additionally, the sdLDL-C level was significantly correlated with AIS severity and bad outcomes. A logistic regression model for assessing the probability of AIS occurrence and a prognostic prediction model were established based on sdLDL-C and other variables.

Conclusions: Elevated levels of sdLDL-C were associated with a higher prevalence of AIS, especially in non-cardioembolic stroke subtypes. After adjustment for other risk factors, sdLDL-C was found to be an independent risk factor for AIS. Also, sdLDL-C level was strongly associated with AIS severity and poor functional outcomes. Logistic regression models for AIS risk and prognosis prediction were established to help clinicians provide better prevention for high-risk subjects and monitor their prognosis.

Key words: sdLDL-C, AIS risk, AIS prognosis, Logistic regression model

\section{Introduction}

Stroke is still the second cause of death and a leading cause of serious disability despite recent advances in diagnosis and treatment ${ }^{1)}$. Over $65 \%$ of stroke deaths occur in the developing countries ${ }^{2)}$. China bears the biggest stroke burden in the world, and stroke becomes the leading cause of death in China $^{3)}$. In China, about $20 \%$ of overall deaths could be attributed to stroke and more than 2 million new

Address for correspondence: Xifa Lan, Qinhuangdao No.1 Hospital, No. 258, Haigang District, Qinhuangdao City, Hebei 441000, P.R. China

E-mail: lanxifa2000@126.com

Received: September 24, 2019 Accepted for publication: January 6, 2020

Copyright@2020 Japan Atherosclerosis Society

This article is distributed under the terms of the latest version of CC BY-NC-SA defined by the Creative Commons Attribution License. 
cases are diagnosed every year ${ }^{4)}$. Additionally, more than 1 million stroke-related deaths occur in China annually, and the burden is increasing as a result of population aging ${ }^{4)}$. Hypertension, dyslipidemia, diabetes mellitus, smoking, and unreasonable dietary habits are the most common risk factors for stroke in China ${ }^{4,5)}$. Better knowledge of stroke risk factors is helpful in reducing the incidence of and mortality associated to stroke in China ${ }^{6}$.

Atherosclerosis plaques are closely associated with ischemic cerebral infarction, which accounts for approximately $80 \%$ of all strokes ${ }^{7}$. Hypercholesterolemia is a well-known vascular risk factor for the development of atherosclerosis and vascular diseases such as stroke. At present, low-density lipoprotein cholesterol (LDL-C) is widely considered to be the primary cause of atherosclerosis and the target to be controlled to reduce atherosclerosis and stroke risk in multiple treatment guidelines ${ }^{8-10)}$. However, a large part of the patients with acute vascular infarction events have normal plasma LDL-C concentrations ${ }^{11)}$. Besides, patients who underwent lipid-lowering treatment and reached a targeted LDL-C level are still at risk of mortality and recurrent cardiovascular and cerebrovascular events ${ }^{12)}$. Based on these findings, lipoprotein studies have recently focused more on subtypes of lipoproteins such as small dense LDL-C (sdLDL-C). The effect of elevated sdLDL-C level is reported to be associated with an increased risk of atherosclerosis in cardiovascular diseases, and sdLDL-C is deemed to be a better predictor of atherosclerosis than total LDL-C level ${ }^{13)}$. For this reason, increased level of sdLDL-C has been accepted as a new risk factor for cardiovascular events by the National Cholesterol Education Program Adult Treatment Panel III ${ }^{14)}$. Nevertheless, few researches have been done on the influence of sdLDLC levels on stroke in Chinese patients due to the complex etiology of stroke, which includes genetic and environmental factors. Therefore, it is necessary to evaluate the relationship between sdLDL-C and stroke in different populations.

In the present study, we examined whether sdLDL-C level worked as a better biomarker for predicting the occurrence, progression, and prognosis of acute ischemic stroke (AIS), and constructed logistic regression models based on potential risk factors involving sdLDL-C as well as other factors to predict the risk and prognosis of AIS, respectively. Based on these findings, we hope to reduce the incidence and burden of stroke in China.

\section{Methods}

Study Popullation

This was a hospital-based follow-up study. A total of 355 patients hospitalized in the Department of Neurology, Xiangyang First people's hospital, Hubei province, China, from October 2016 to April 2018 with the diagnosis of first-ever AIS were recruited (Supplementary Fig. 1). All patients were followed for 3 months after AIS onset. In the current study, participants were excluded for the following reasons: (1) having past history of cerebral infarction, (2) severe cardiovascular disease, such as coronary heart disease and myocardial infarction, (3) bleeding or other pathological brain diseases, such as head trauma, primary and metastatic neoplasms, abscesses, or other non-ischemic cerebral diseases diagnosed by computed tomography and/or magnetic resonance imaging, (4) transient ischemic attack; and (5) being lost to followup. We enrolled 171 non-AIS subjects with no ischemic cerebrovascular events (including vertigo, syncope, migraine, trigeminal neuralgia, and epilepsy) and people without diagnosed neurological diseases who had physical examination in the department of neurology. All non-AIS subjects passed a medical evaluation including a complete physical examination and radiological examination to exclude the existence of stroke, and were deemed as the control group in this study. The study was approved by the Research Ethics Committee of Hubei Medical College and informed consent forms were filled out. The disease of AIS was diagnosed clinically; the ischemic lesion was measured by magnetic resonance imaging, computed tomography angiography, and digital subtraction angiography. Stroke severity was assessed based on the National Institutes of Health Stroke Scale (NIHSS) and the modified Rankin Scale (mRS) ${ }^{15,16)}$. Clinical information including age, gender, body mass index (BMI), blood glucose and lipid levels, history of hypertension or diabetes mellitus, smoking or drinking habits, and statin therapy records were recorded.

\section{NIHSS and mRS at Admission}

The NIHSS is a measure to evaluate impairments caused by acute strokes and numerical values related to multiple aspects of neuromotoxic functions including language, motor, sensory, consciousness, speech, etc. The scores range from 0 to a maximum of 42, which represents different impairment severities including no impairment (0), mild (1-4), moderate $(5-15)$, and moderate-severe $(16-42)^{17)}$. The mRS is a 6-point scale that focuses on neurological functions and individual living capacity in post-stroke patients. The scores range from 0 to 5 and describe progressive 
severity. A score of 6 represents death ${ }^{18)}$.

\section{Stroke Subtypes}

The TOAST criteria was used to classify the subtypes of AIS ${ }^{19)}$ : (1) large-artery atherosclerosis (LAA), (2) lacunar stroke or small-artery occlusion (SAO), (3) cardioembolism (CE), (4) stroke of other determined cause (ODC), and (5) stroke of undetermined cause.

\section{Stroke Outcomes}

Stroke outcomes were assessed at 3 months after stroke. Mortality was defined as all-cause death before the follow-up time after stroke. Dependency was defined as $\mathrm{mRS}>2$ and independency was defined as $m R S \leq 2^{20}$. These assessments were done through telephone calls or face-to-face interviews.

\section{Laboratory Tests}

Blood glucose, total cholesterol (TC), triglyceride (TG), and lipoproteins were measured in all patients using fasting blood samples (8-h overnight) drawn into serum separator tubes and centrifuged at 3,000 $\mathrm{rpm}$ for $15 \mathrm{~min}$ at $4{ }^{\circ} \mathrm{C}$ to separate the serum. The serum parameters were determined using an ARCHITECT c16000 automatic biochemical analyzer (Abbott Laboratories, Abbott Park, IL, USA) according to the manufacturer's instructions. The analysis of lipoprotein subfractions including low-density lipoprotein (LDL) 1, 2, 3, 4, 5, 6, and 7 were detected by the Lipoprint LDL system (Quantimetrix Corp., USA). LDL comprises seven subclasses including LDL 1 to LDL 7 in gradient gel electrophoresis ${ }^{21)}$, which differ in size, density, physicochemical composition, and metabolic behavior. LDL 1 and 2 are defined as large LDLs, and LDL 3 to 7 are defined as sdLDLs according to their size and density ${ }^{13)}$.

\section{Logistic Regression Model}

In the machine learning framework, the logistic regression models of the score were calculated using the risk factors to predict stroke risk and outcome. In brief, the logistic score which predicted the probability of risk according to the logistic regression equation could be achieved with the following formula:

$$
\text { Predicted risk probability }=\frac{\mathrm{e}^{(\beta 0+\Sigma \beta i \mathrm{X})}}{1+\mathrm{e}^{(\beta 0+\Sigma \beta \mathrm{X} X)}}
$$

In brief, $\beta 0$ is the constant of the logistic regression formula and $\beta i$ is the coefficient of the variable $X i$ in the logistic regression formula. $X i=1$ means that a categorical risk factor is present and 0 means that a categorical risk factor is absent. The logistic score has a good application value for individual risk prediction in very high-risk patients, which contributes to further research in risk field ${ }^{22)}$.

\section{Statistical Analysis}

All data were analyzed using Python software (Version 3.6). Categorical variables were presented as numbers and proportions (\%), continuous variables as mean \pm standard deviation or minimal and maximal values. The Mann-Whitney $U$ test and the chisquared test were used to analyze intra-group or intergroup differences. The Kruskal-Wallis H test was performed to detect differences between subgroups of different outcomes. Correlation analysis including the relationship of clinical values and stroke subtypes, severity, and outcomes were performed using the Pearson correlation method. XGBoost Classifier decision trees model (XGBoost 0.81), Random Forest Classifier, Logistic Regression, and AdaBoost Classifier were performed to predict the AIS risk among patients. Data of the models were presented as receiver operating characteristics (ROC) curves and area under the curve (AUC). The closer AUC approaches 1, the better the model performance. Logistic regression models were constructed for the prediction of the risk and prognosis of AIS. To identify the most parsimonious predictive model, variables unrelated to the risk or outcomes and whose removal had little impact on model fit were removed. A $P$-value $<0.05$ was considered to be statistically significant.

\section{Results}

\section{Clinical Characteristics of Participants}

The mean age of the patients and controls was $64.26 \pm 10.73$ and $62.95 \pm 11.91$ years old with a male:female ratio of 60.28:39.72 and 57.89:42.11, respectively. Overall, all the recruited participants had a normal BMI. The median NIHSS was 6.25 (range, 0-33), and the mean mRS score was 1.06 (range, 0-5) at admission. A higher prevalence of hypertension $(72.11 \%$ vs. $59.65 \%)$ and diabetes $(26.76 \%$ vs. $19.30 \%)$ were observed in the AIS group than in the control group. The prevalence of smoking and drinking was comparable in patients with and without AIS $(P>0.05)$. There were significant differences in the levels of glucose, TG, and LDL subtypes between the two groups which are listed in Table 1 . The serum sdLDL-C levels in the group without AIS were 13.21 $\pm 5.97 \mathrm{mg} / \mathrm{dL}$, which were significantly lower than those in the AIS group $(24.89 \pm 19.97 \mathrm{mg} / \mathrm{dL})$. However, the TC, total LDL-C, and high-density lipoprotein cholesterol (HDL-C) levels did not differ between the two groups $(P>0.05)$, and the values of TC, TG, LDL-C, and HDL-C in the two groups were all within the normal reference ranges. There was no significant difference in the proportion of patients receiving statin therapy between the AIS and the control 
Table 1. General characteristics of the participants

\begin{tabular}{|c|c|c|c|}
\hline Variable & Patients $(n=355)$ & Controls $(n=171)$ & $P$ value \\
\hline Age (years) ${ }^{*}$ & $64.26 \pm 10.73$ & $62.95 \pm 11.91$ & 0.160 \\
\hline Male / Female sex & $214(60.28) / 141(39.72)$ & $99(57.89) / 72(42.11)$ & 0.301 \\
\hline BMI $\left(\mathrm{kg} / \mathrm{m}^{2}\right)^{*}$ & $23.53 \pm 2.79$ & $23.60 \pm 2.60$ & 0.155 \\
\hline NIHSS ${ }^{*}$ & $6.25(0-33)$ & l & \\
\hline $\mathrm{mRS}^{*}$ & $1.06(0-5)$ & l & \\
\hline \multicolumn{4}{|l|}{ TOAST } \\
\hline LAA & $186(52.39)$ & l & \\
\hline SAO & $157(44.23)$ & l & \\
\hline CE & $8(2.25)$ & l & \\
\hline ODC & $4(1.13)$ & l & \\
\hline UC & $0(0.00)$ & l & \\
\hline Hypertension & $256(72.11)$ & $104(59.65)$ & 0.002 \\
\hline Diabetes & $95(26.76)$ & $33(19.30)$ & 0.031 \\
\hline Smoker & $107(30.14)$ & $49(28.65)$ & 0.364 \\
\hline Drinker & $92(25.92)$ & $42(24.56)$ & 0.370 \\
\hline Glucose $(\mathrm{mmol} / \mathrm{L})^{*}$ & $6.71 \pm 2.30$ & $6.05 \pm 1.65$ & 0.000 \\
\hline $\mathrm{TC}(\mathrm{mmol} / \mathrm{L})^{*}$ & $3.83 \pm 0.91$ & $0.85 \pm 0.85$ & 0.175 \\
\hline $\mathrm{TG}(\mathrm{mmol} / \mathrm{L})^{*}$ & $1.42 \pm 1.09$ & $1.30 \pm 0.74$ & 0.034 \\
\hline LDL-C $(\mathrm{mmol} / \mathrm{L})^{*}$ & $2.18 \pm 0.65$ & $2.15 \pm 0.76$ & 0.349 \\
\hline LDL1-C (mg/dl)* & $18.37 \pm 8.04$ & $26.32 \pm 7.67$ & 0.000 \\
\hline LDL2-C (mg/dl)* & $21.09 \pm 6.84$ & $24.13 \pm 7.89$ & 0.000 \\
\hline LDL3-C (mg/dl)* & $12.30 \pm 7.01$ & $9.02 \pm 3.65$ & 0.000 \\
\hline LDL4-C (mg/dl)* & $6.50 \pm 6.43$ & $2.99 \pm 2.07$ & 0.000 \\
\hline LDL5-C (mg/dl)* & $2.82 \pm 4.52$ & $1.11 \pm 1.57$ & 0.000 \\
\hline LDL6-C $(\mathrm{mg} / \mathrm{dl})^{*}$ & $0.90 \pm 1.98$ & $0.08 \pm 0.32$ & 0.000 \\
\hline LDL7-C (mg/dl) & $1.38 \pm 3.19$ & $0.02 \pm 0.13$ & 0.000 \\
\hline sdLDL-C (mg/dl) ${ }^{*}$ & $24.89 \pm 19.97$ & $13.21 \pm 5.97$ & 0.000 \\
\hline HDL-C $(\mathrm{mg} / \mathrm{dl})^{*}$ & $41.86 \pm 12.59$ & $43.31 \pm 11.25$ & 0.089 \\
\hline Statin therapy & $13(3.66)$ & $10(5.85)$ & 0.122 \\
\hline
\end{tabular}

Categorical variables presented as numbers and proportions (\%), continuous variables as mean \pm standard deviation (SD), minimal and maximal values *

BMI, body mass index

groups (3.66\% and 5.85\%, respectively).

\section{Correlation between Clinical Variables and AIS Risk}

Pearson correlation analysis was conducted to determine the correlation of clinical risk factors and the presence of AIS (Fig. 1). There is a positive and significant correlation between AIS risk and the levels of LDL subtypes 3-7 (sdLDL-C) $(r>0.2, P=0.000)$, glucose $(r=0.14, P=0.001)$, and presence of hypertension $(\mathrm{r}=0.13, P=0.004)$. A significant inverse correlation between AIS risk and LDL1-C $(\mathrm{r}=-0.43$, $P=0.000)$ and LDL2-C $(\mathrm{r}=-0.19, P=0.000)$ was found in the study. No significant correlation was observed between AIS risk and other baseline variables of the participants.
Multivariable Logistic Regression Analysis of Risk Factors for AIS

The etiology of stroke is complex, involving both environmental and genetic risk factors. Factors, such as LDL-C, glucose levels, diabetes, and hypertension are considered traditional risk factors for atherosclerosis. In addition to classic risk factors, sdLDL-C is also considered to be an independent risk factor for developing ischemic strokes and has drawn more and more attention in recent years ${ }^{23-25)}$. In this study, the multivariable logistic regression used AIS as a response variable, and risk factors as explanatory variables. We found that three variables (sdLDL-C, glucose, and hypertension) with an odds ratio (OR) greater than 1 and a $P$-value less than 0.05 were deemed as risk factors (Table 2). The sdLDL-C level was proved to be an independent risk factor for AIS $(P=0.004)$ in the 


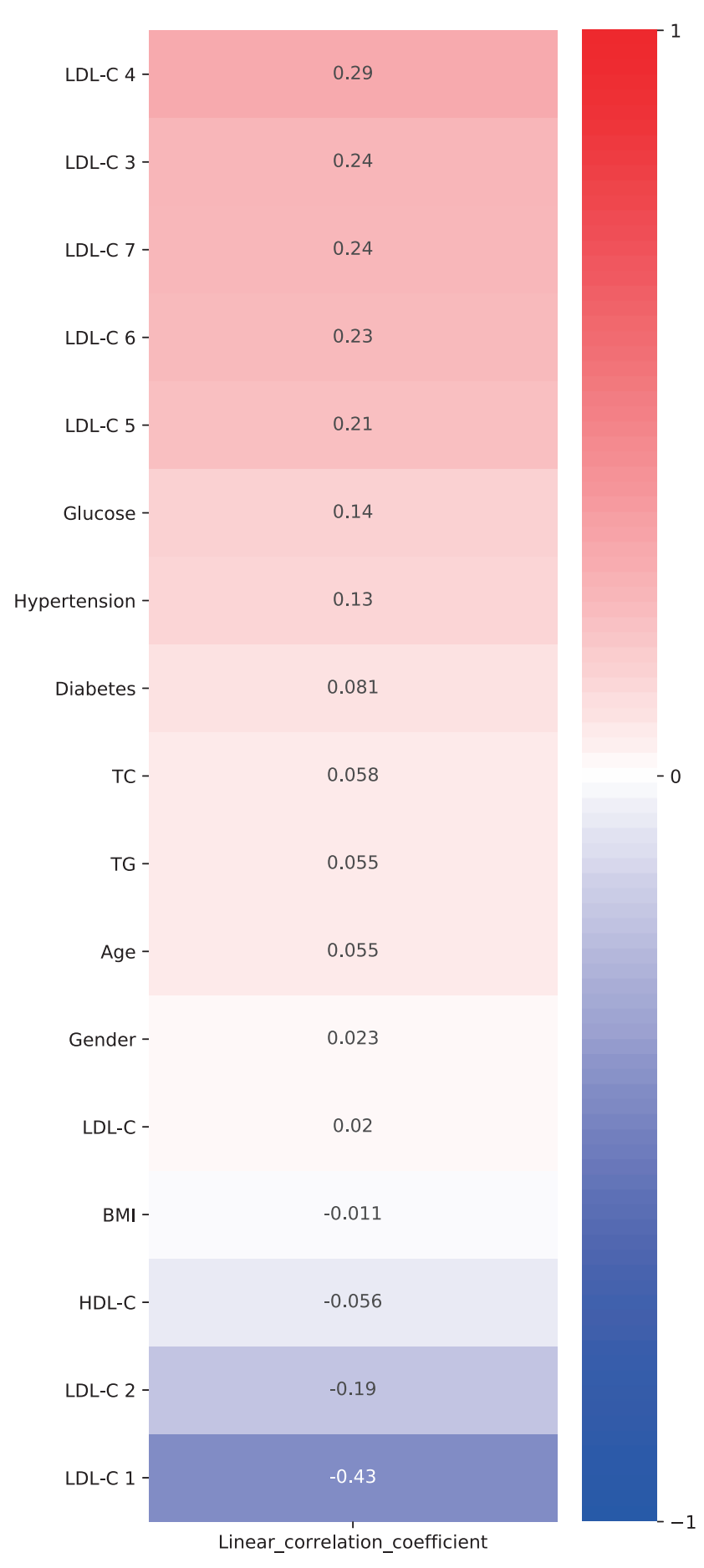

Fig. 1. Pearson correlation between clinical characteristics and AIS risk

basic model (base model; OR, 1.04; 95\% confidence interval [CI], 1.012-1.066) and after additional adjustment for diabetes mellitus, hypertension, glucose level, and other lipid risk factors (model 1) (OR, 1.02; 95\% CI, 1.015-1.030) (Table 3). Pearson correlation analysis was used to evaluate the correlation between sdLDL-C and other clinical indexes. There was a significant positive correlation between sdLDL$\mathrm{C}$ and the levels of blood glucose, TC, TG, and LDL-C in AIS patients $(r>0.1, P<0.01)$ (Supplementary Table 1).

\section{PredictiveValue of sdLDL-C for AIS Risk}

To further evaluate the predictive values of TG, TC, HDL-C, LDL-C, and non-HDL-C in patients with AIS, the ROC curves and AUCs were created and depicted (Fig.2). The ROC curve revealed that the best cut-off value of the sdLDL-C to predict AIS was $23 \mathrm{mg} / \mathrm{dL}$ with $34.65 \%$ sensitivity and $96.49 \%$ specificity. The AUC of sdLDL-C for AIS was 0.665 , which was higher than that of other serum lipids. This indicated that serum sdLDL-C level had a better risk prediction for AIS than other lipid parameters, including LDL-C.

\section{Relationship of Lipids with AIS Subtypes}

To further study association between AIS subtypes and clinical characteristics including sdLDL-C, we adopted the following analysis. The ODC group was younger than other subtype groups. The scores of NIHSS and mRS were lower in the ODC group. The sdLDL-C level in the ODC group was the lowest among all the groups. There was no significant difference among the AIS subtypes in terms of LDL1-C and LDL2-C. There was also no significant difference when the AIS subtypes were compared with each other in terms of total LDL-C, HDL-C, and TC values (Supplementary Table 2).

\section{Correlation between Lipids and AIS Severity}

There was a positive correlation between the levels of sdLDL-C, LDL-C, TC, glucose, and stroke severity $(r>0.15, P<0.01)$. However, the LDL1-C level and stroke severity were negatively correlated $(\mathrm{r}=$ -0.24, $P=0.000$ ) (Fig. 3). AIS patients with mild ( $n=$ $154)$, moderate $(n=111)$, and severe $(n=51)$ stroke evaluated by NIHSS were are listed in Table 4 . The more severe the stroke, the higher the values of sdLDL-C, LDL-C, TC, and TG $(P<0.05)$. Furthermore, only sdLDL-C and TC showed statistical difference between asymptomatic and mild patients $(P<$ $0.05)$.

\section{Influence of Lipids on Prognosis of Stroke}

The mortality, dependency, and independency rates during follow-up were $1.13 \%, 13.24 \%$, and $85.63 \%$ at 3 months, respectively. The TC, TG, LDL$\mathrm{C}$, and sdLDL-C levels were closely associated with the stroke outcomes (Table 5). Compared with the independency group, higher levels of TC, TG, and 
Table 2. Multivariable logistic regression analysis of risk factors for stroke

\begin{tabular}{lccc}
\hline Variables & OR value & $95.0 \%$ CI for OR & $P$ \\
\hline TC & 1.909 & $1.091-3.340$ & 0.023 \\
SdLDL-C & 1.039 & $1.012-1.066$ & 0.004 \\
Glucose & 1.115 & $0.964-1.292$ & 0.144 \\
Hypertension & 1.699 & $1.087-2.659$ & 0.020 \\
\hline
\end{tabular}

CI, confidence interval

OR, odds ratio

Table 3. Logistic regression analysis for independent association between sdLDL-C and the onset of AIS

\begin{tabular}{lcccccc}
\hline & B-value & SE & Wals $\chi^{2}$ & OR value & $95.0 \%$ CI for OR & $P$ \\
\hline Model & 0.0379 & 0.013 & 8.038 & 1.04 & $1.012-1.066$ & 0.005 \\
Model 1 & 0.0229 & 0.004 & 35.026 & 1.02 & $1.015-1.030$ & 0.000 \\
\hline
\end{tabular}

CI, confidence interval

Model 1 adjusted for diabetes mellitus, hypertension, glucose, TC, TG, LDL-C, LDL1-C and LDL2-C

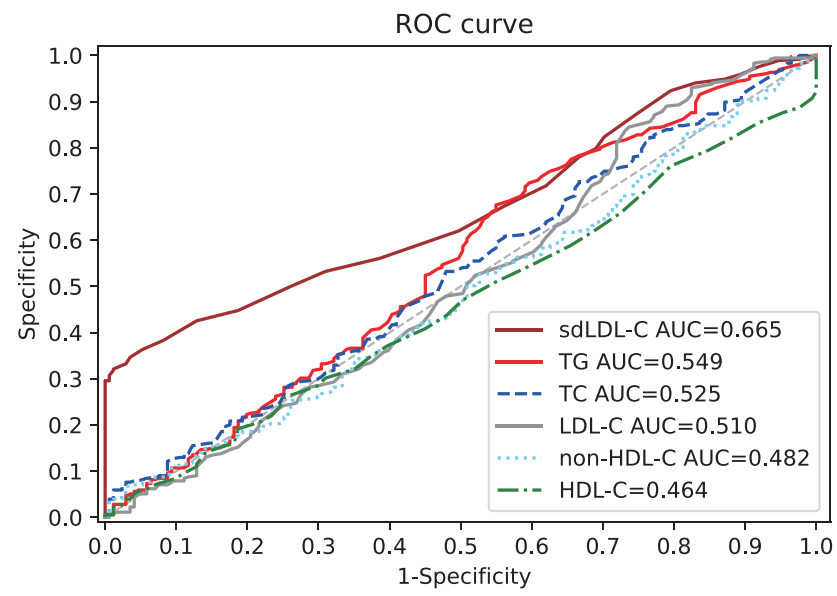

Fig. 2. Predictive values of serum lipids for AIS risk

The ROC curves and AUCs regarding risk are depicted in Fig. 2. AUCs: 0.665 for sdLDL-C, 0.549 for TG, 0.525 for TC, 0.510 for LDL-C, 0.482 for non-HDL-C, and 0.464 for HDL-C.

LDL-C were observed in the dependency group. sdLDL-C was the only index that was positively correlated with clinical outcomes. The sdLDL-C level of the mortality, dependency, independency groups decreased by group, suggesting that sdLDL-C is a risk factor for adverse outcome of stroke. In addition, there was a higher risk of mortality and dependency in women than in men at 3 months after stroke. NIHSS and mRS scores and glucose level were significantly associated with quality of life at 3 months after stroke (Supplementary Table 3).

Logistic Regression Models for Predicting the Risk and Prognosis of AIS

Several typical and recent artificial intelligence algorithms were assessed in the context of AIS, and the logistic regression became our base model (AUC= $0.77 \pm 0.13$ ) (Supplementary Fig. 2). A logistic regression model for assessing the probability of AIS occurrence and effectively distinguishing high-risk from low-risk patients with cerebrovascular diseases was built, with an AUC of 0.813 (Fig.4). Conventional non-lipid and lipid risk factors (history of diabetes, hypertension, glucose, TC, TG, LDL-C, LDL1-C, LDL2-C, and sdLDL-C levels) were included in the model. A prognostic prediction model was established to predict the probability of improvement 90 days after AIS. The variables that remained in the regression model were age, history of diabetes, hypertension, NIHSS, glucose, TC, TG, LDL-C, LDL1-C, LDL2-C, and sdLDL-C levels. The AUC of this model was 0.926 (Fig. 5). No matter for sdLDL-C, NIHSS, age, and other factors, the effect of model on prognosis prediction based on multiple variables was better than that of single-risk factor (Supplementary Fig. 3). The intercept, 95\% CI, and slope are shown in Supplementary Table 4.

\section{Discussion}

Stroke is the first cause of death and the second cause of disability in adults. AIS accounts for about $85 \%$ of the stroke cases. Because of the high incidence, mortality, and disability associated with AIS, it brings a heavy burden to the patients, society, and country ${ }^{26)}$. Thus, the establishment of early screening methods for a high-risk population can provide early clinical guidance and intervention to avoid loss caused by stroke.

Cholesterol has been shown to be a useful bio- 


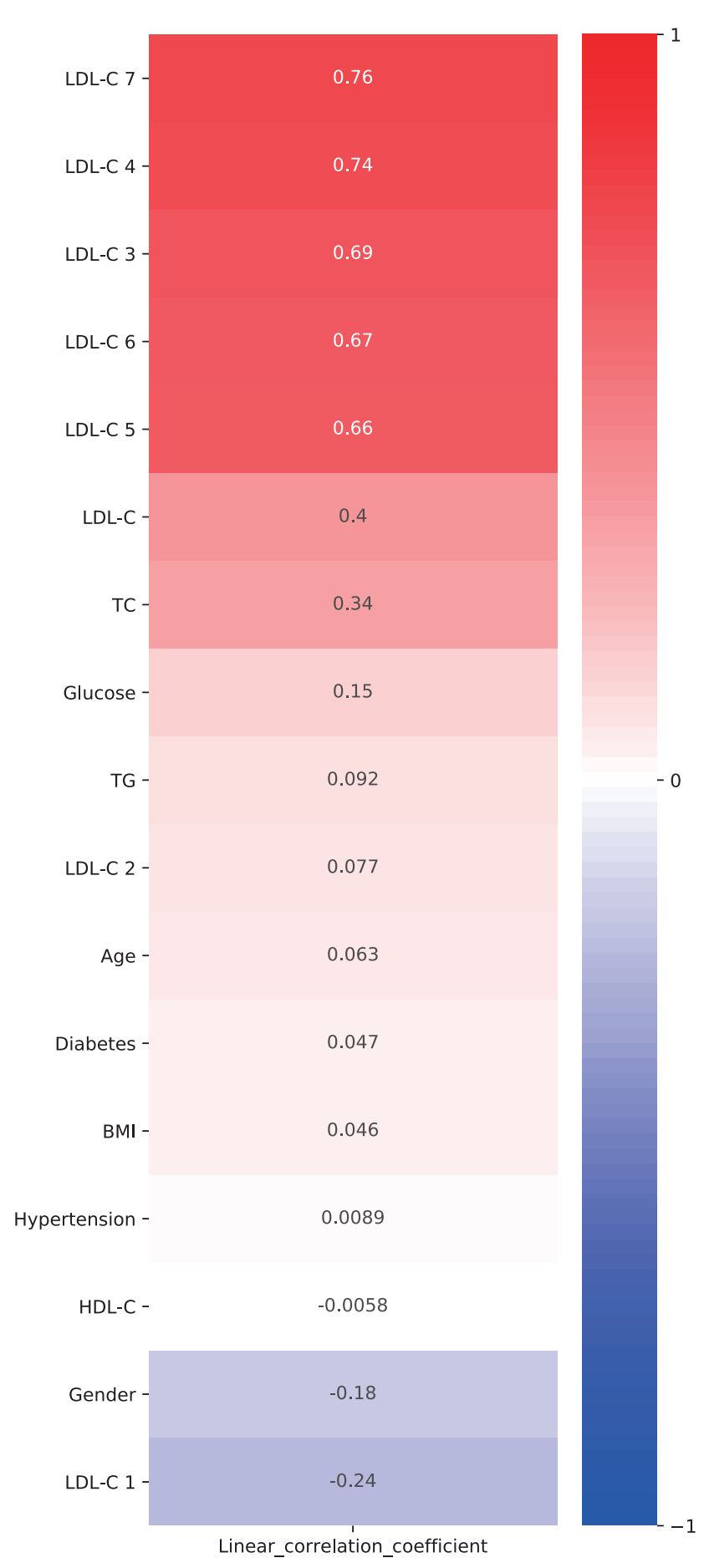

Fig.3. Pearson correlation between clinical characteristics and AIS severity

marker to predict ischemic stroke risk. The role of serum LDL-C level and atherosclerosis has been revealed in the following years ${ }^{27)}$. However, a high proportion of patients with atherosclerosis or acute vascular events has a normal range of plasma cholesterol and LDL-C ${ }^{11)}$, implying that the increased risk of atherosclerosis of vascular diseases is due to other lipoproteins. The same relationship between nonHDL-C and ischemic stroke was found. The National Cholesterol Education Program Adult Treatment Panel III has recommended LDL-C as the primary target of cholesterol-lowering therapy, with nonHDL-C as the secondary target ${ }^{8)}$. However, increasing evidence suggests that non-HDL-C is a better predictor than LDL-C ${ }^{28,29)}$. Recently, studies have confirmed that LDL-Cs consist of a series of particles with different sizes, densities, chemical properties, and functions ${ }^{30)}$. Thus, LDL-Cs are classified into two types, A (subtype 1-2, large and buoyant LDL-C) and B (subtype 3-7, sdLDL-C). Previous studies demonstrated that VLDL, IDL1-3, and sdLDL-C act as atherogenic lipoprotein profiles ${ }^{31,32)}$. sdLDL-C is significantly associated with atherosclerosis and carotid plaques among them ${ }^{33,34)}$. Though sdLDL-C has been considered a better biomarker to predict cardiovascular and cerebrovascular diseases than LDL-C in the recent years, the relationship between serum sdLDL-C levels and AIS risk are equivocal. In this study, a positive and significant correlation between AIS risk and the levels of sdLDL-C and glucose and presence of hypertension was found. Besides, there was a significant positive correlation between sdLDL-C levels and serum lipids including TC, TG, and LDL-C. Though LDL-C has long been identified as a risk factor for AIS, TC, TG, LDL-C, and HDL-C in both groups were within the normal reference range in both AIS patients and controls, suggesting that it is not the best biomarker for AIS prediction. Multivariable logistic regression confirmed that sdLDL-C was an independent variable of AIS after additional adjustment for diabetes mellitus, hypertension, glucose level, and other lipid risk factors. Additionally, the sdLDL-C AUC of AIS was superior to that of other lipids, which indicated that serum sdLDL-C level had a better risk prediction for AIS than other lipids, including LDL-C. Considering all the evaluated values of clinical parameters in stroke, a model was constructed to predict the AIS risk in patients at high risk. sdLDL-C, history of diabetes, hypertension, glucose level, TG, TC, LDL-C, LDL1-C, and LDL2-C were input in the model as variables, and the corresponding risk range can be output. Moreover, an AUC $>0.8$ in the training set suggested that the sdLDL-C plus other factors could serve as a superior evaluation system to reflect the AIS risk.

Limited studies exploring the relationship between LDL subclasses and AIS subtypes have been done. The atherogenic effect of sdLDL particles on carotid atherosclerosis is well known ${ }^{35)}$. Higher levels of sdLDL-C and LDL-C were found in LAA, SAO, 
Table 4. Correlation between biochemical parameters and stroke severity

\begin{tabular}{lcccc}
\hline Variables & None $(n=39)$ & Mild $(n=154)$ & Moderate-severe $(n=111)$ & Severe $(n=51)$ \\
\hline TC $(\mathrm{mmol} / \mathrm{L})$ & $3.33 \pm 0.58^{\mathrm{a}}$ & $3.59 \pm 0.77^{\mathrm{b}}$ & $4.05 \pm 0.95^{\mathrm{c}}$ & $4.46 \pm 0.93^{\mathrm{d}}$ \\
TG $(\mathrm{mmol} / \mathrm{L})$ & $1.40 \pm 0.88^{\mathrm{a}}$ & $1.28 \pm 1.32^{\mathrm{a}}$ & $1.50 \pm 0.90^{\mathrm{b}}$ & $1.69 \pm 0.75^{\mathrm{c}}$ \\
LDL-C $(\mathrm{mmol} / \mathrm{L})$ & $1.87 \pm 0.50^{\mathrm{a}}$ & $1.95 \pm 0.54^{\mathrm{a}}$ & $2.35 \pm 0.64^{\mathrm{b}}$ & $2.72 \pm 0.63^{\mathrm{c}}$ \\
LDL1-C $(\mathrm{mg} / \mathrm{dl})$ & $19.82 \pm 6.75^{\mathrm{a}}$ & $20.53 \pm 8.68^{\mathrm{a}}$ & $16.61 \pm 7.14^{\mathrm{b}}$ & $14.61 \pm 6.36^{\mathrm{c}}$ \\
LDL2-C $(\mathrm{mg} / \mathrm{dl})$ & $19.82 \pm 6.12^{\mathrm{a}}$ & $20.34 \pm 6.50^{\mathrm{a}}$ & $22.09 \pm 7.40^{\mathrm{b}}$ & $22.16 \pm 6.59^{\mathrm{b}}$ \\
sdLDL-C $(\mathrm{mg} / \mathrm{dl})$ & $8.87 \pm 4.13^{\mathrm{a}}$ & $12.46 \pm 6.14^{\mathrm{b}}$ & $26.97 \pm 11.26^{\mathrm{c}}$ & $63.16 \pm 15.60^{\mathrm{d}}$ \\
HDL-C $(\mathrm{mg} / \mathrm{dl})$ & $38.08 \pm 8.83^{\mathrm{a}}$ & $42.26 \pm 13.58^{\mathrm{ab}}$ & $43.24 \pm 13.3^{\mathrm{b}}$ & $40.51 \pm 9.22^{\mathrm{ab}}$ \\
\hline
\end{tabular}

Continuous variables as mean \pm standard deviation $(\mathrm{SD})$

Groups that are not labeled with any of the same letters $(a, b, c)$ indicate statistically significant at 0.05 . Mann whitney $U$ test for continuous values

Table 5. Correlation between biochemical parameters and stroke outcomes

\begin{tabular}{lcccc}
\hline Variables & Mortality $(n=4)$ & Dependency $(n=47)$ & Independency $(n=304)$ & $P$ value \\
\hline TC $(\mathrm{mmol} / \mathrm{L})$ & $4.22 \pm 0.90$ & $4.45 \pm 1.07$ & $3.73 \pm 0.84$ & 0.000 \\
TG $(\mathrm{mmol} / \mathrm{L})$ & $1.31 \pm 0.38$ & $1.61 \pm 0.81$ & $1.39 \pm 1.13$ & 0.025 \\
LDL-C $(\mathrm{mmol} / \mathrm{L})$ & $2.54 \pm 0.74$ & $2.67 \pm 0.73$ & $2.10 \pm 0.60$ & 0.000 \\
LDL1-C $(\mathrm{mg} / \mathrm{dl})$ & $13.00 \pm 3.54$ & $16.02 \pm 7.56$ & $18.81 \pm 8.07$ & 0.016 \\
LDL2-C $(\mathrm{mg} / \mathrm{dl})$ & $18.75 \pm 4.44$ & $21.19 \pm 6.39$ & $21.11 \pm 6.92$ & 0.734 \\
sdLDL-C $(\mathrm{mg} / \mathrm{dl})$ & $63.50 \pm 40.02$ & $50.77 \pm 22.86$ & $19.21 \pm 14.48$ & 0.000 \\
HDL-C $(\mathrm{mg} / \mathrm{dl})$ & $48.25 \pm 7.95$ & $41.94 \pm 11.03$ & $41.76 \pm 12.84$ & 0.367 \\
\hline
\end{tabular}

Continuous variables as mean \pm standard deviation (SD)

The Kruskal-Wallis $\mathrm{H}$ test for continuous values

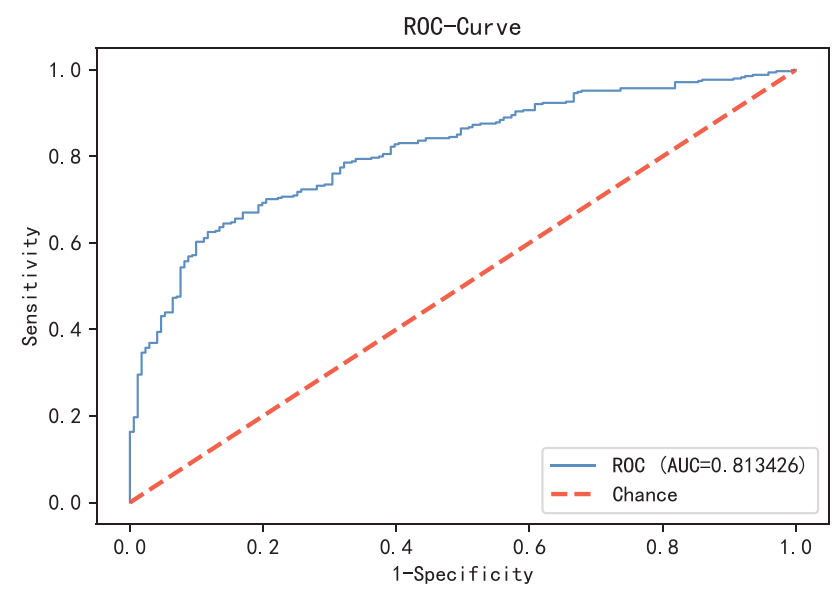

Fig.4. An established model for prediction of AIS risk

and $\mathrm{CE}$ subgroups maybe based on pathological changes. In this study, sdLDL-C was found to be significantly lower in the ODC subgroup compared to the other groups. Synchronized with sdLDL-C were the NIHSS and mRS scores in ODC patients, suggesting a better outcome of the ODC group.

The NIHSS is widely used to evaluate the severity of stroke. Overall, patients with high NIHSS scores have more severe symptoms in the acute phase, often associated with massive cerebral infarction. The risk

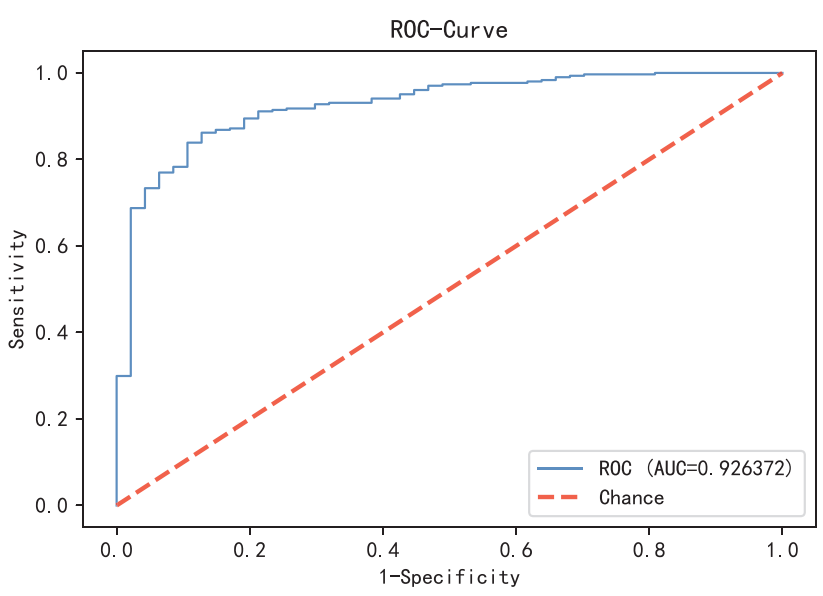

Fig. 5. An established model for prediction of AIS outcomes

factors for plaque formation in AIS patients is complex. There are few studies about the association between the concentration of lipoprotein subfractions and stroke severity. In this study, NIHSS and mRS were used to assess atherosclerotic severity. Then, its correlation with lipoprotein levels was conducted. As expected, the current study demonstrated that the sdLDL-C levels were positively correlated with stroke severity, and this correlation is the highest compared to other clinical risk factors. The more severe the 
stroke, the higher the sdLDL-C, LDL-C, TC, and TG values. However, only the sdLDL-C and TC levels in the asymptomatic and mild patients had statistical differences. sdLDL-C becomes a much stronger and more significant diagnostic value in detection and grading of AIS.

The relationships between plasma lipoprotein levels and stroke outcome have remained controversial, though some researches have made relevant studies around this topic. A recent study suggested that poor outcome (NIHSS score $>10$ or death) for patients with AIS was related to elevated LDL-C levels at admission ${ }^{36)}$. A significant positive correlation was found between higher LDL-C levels and higher mortality rates at both 12 and 36 months after stroke ${ }^{37)}$. Contrarily, another article reported no statistical association between LDL-C levels and mortality or dependency percentage at 3 months after stroke in China ${ }^{38)}$. Statin therapy has become the most important measure to prevent stroke, and it is believed that a decrease in the cholesterol levels due to statin treatment can reduce the incidence of stroke in high-risk populations ${ }^{39)}$. However, in this study, the correlation between the history of statin use and stroke outcomes due to the very low number of deaths in the mortality group is questionable. Considering the joint evaluated values of these parameters in stroke outcome, a model was constructed to predict the stroke outcome in patients with AIS. sdLDL-C, age, history of diabetes, hypertension, glucose, TG, TC, LDL-C, LDL1-C, LDL2-C, and NIHSS score were input in the model as variables, and the corresponding probability of improvement can be output, predicting 3-month prognosis of patients with AIS in China. An AUC > 0.9 of the model exhibited its advantage and all variables in the model are conventional information, which is easy for clinicians to use.

\section{Limitations}

A small sample size from a single neurological hospital in China is the main limitation of this study, the small sample size is easy to cause the deviation of grouping. Second, the number of cardioembolisms and other stroke subtypes was very small, which may have affected the statistical power of the study. Third, data on sdLDL-C at follow-up was lacking, which might have affected the evaluation of the effect of sdLDL-C on stroke outcome. In future studies, it is necessary to further expand the sample size, especially for cardiogenic and other types of stroke patients, to study the role of sdLDL-C level in the occurrence and prognosis of AIS.

\section{Conclusion}

The current study demonstrated that sdLDL-C is an independent risk factor for AIS, especially for non-cardioembolic stroke. The predictive value of sdLDL-C was better than that of other lipids. Besides, sdLDL-C levels were associated with the severity and prognosis of AIS, contributing to guiding prompt and correct treatment and secondary prevention for patients. The models based on sdLDL-C and other variables for AIS risk and prognosis were established to monitor patients who are at high risk for acute vascular events and monitor their stroke prognosis. Therefore, controlling the levels of sdLDL-C is of great significance in preventing the occurrence and development of stroke.

\section{Statement of Authorship}

Peiyang Zhou and Xifa Lan are responsible for the study design; Wang-yang $\mathrm{Xu}$ is responsible for analyzing the data and writing of the manuscript; Jincheng Liu, Lingyun Wang, Wenmin Feng, Zhihua Cao, Pu Wang, Guangzhi, Liu, Chenglin Sun and Yan Shen are responsible for collection of samples and questionnaires; Lijun Wang and Jiahuan $\mathrm{Xu}$ are responsible for dealing with the data and constructing the models; Peng Meng and Ziwei Li are responsible for the calibration of manuscript; All authors listed above were participated in processing patient information. All authors approved the final version of the manuscript.

\section{Conflict of Interest}

The authors declare no conflict of interest.

\section{Funding}

This research was supported by the Major Projects of Special Development Funds in Zhangjiang National Independent Innovation Demonstration Zone, Shanghai ZJ2017-ZD-012 .

\section{Acknowledgements}

We are particularly grateful to all the participants in this research. We thank for the efforts of Xiangyang First people's hospital and we appreciate the help from Shanghai Zhangjiang Center for Translational Medicine and Shanghai Zhangjiang Institute of Medical Innovation. 


\section{References}

1) Feigin VL, Forouzanfar MH, Krishnamurthi R, Mensah GA, Connor M, Bennett DA, Moran AE, Sacco RL, Anderson L, Truelsen T, O’Donnell M, Venketasubramanian N, Barker-Collo S, Lawes CM, Wang W, Shinohara Y, Witt E, Ezzati M, Naghavi M, Murray C, Global Burden of Diseases I, Risk Factors $S$ and the GBDSEG: Global and regional burden of stroke during 1990-2010: findings from the Global Burden of Disease Study 2010. Lancet, 2014; 383: 245-254

2) Bonita R, Mendis S, Truelsen T, Bogousslavsky J, Toole J and Yatsu F: The global stroke initiative. Lancet Neurol, 2004; 3: 391-393

3) Wu S, Wu B, Liu M, Chen Z, Wang W, Anderson CS, Sandercock P, Wang Y, Huang Y, Cui L, Pu C, Jia J, Zhang T, Liu X, Zhang S, Xie P, Fan D, Ji X, Wong KL, Wang L and China Stroke Study C: Stroke in China: advances and challenges in epidemiology, prevention, and management. Lancet Neurol, 2019; 18: 394-405

4) Wang W, Jiang B, Sun H, Ru X, Sun D, Wang L, Wang L, Jiang Y, Li Y, Wang Y, Chen Z, Wu S, Zhang Y, Wang D, Wang Y, Feigin VL and Investigators NE-C: Prevalence, Incidence, and Mortality of Stroke in China: Results from a Nationwide Population-Based Survey of 480687 Adults. Circulation, 2017; 135: 759-771

5) Guan T, Ma J, Li M, Xue T, Lan Z, Guo J, Shen Y, Chao B, Tian G, Zhang Q, Wang L and Liu Y: Rapid transitions in the epidemiology of stroke and its risk factors in China from 2002 to 2013. Neurology, 2017; 89: 53-61

6) Pandian JD, Gall SL, Kate MP, Silva GS, Akinyemi RO, Ovbiagele BI, Lavados PM, Gandhi DBC and Thrift AG: Prevention of stroke: a global perspective. Lancet, 2018; 392: 1269-1278

7) Mughal MM, Khan MK, DeMarco JK, Majid A, Shamoun F and Abela GS: Symptomatic and asymptomatic carotid artery plaque. Expert Rev Cardiovasc Ther, 2011; 9: $1315-1330$

8) Expert Panel on Detection E and Treatment of High Blood Cholesterol in A: Executive Summary of The Third Report of The National Cholesterol Education Program (NCEP) Expert Panel on Detection, Evaluation, And Treatment of High Blood Cholesterol In Adults (Adult Treatment Panel III). JAMA, 2001; 285: 2486-2497

9) Goldstein LB, Bushnell CD, Adams RJ, Appel LJ, Braun LT, Chaturvedi S, Creager MA, Culebras A, Eckel RH, Hart RG, Hinchey JA, Howard VJ, Jauch EC, Levine SR, Meschia JF, Moore WS, Nixon JV, Pearson TA, American Heart Association Stroke C, Council on Cardiovascular N, Council on E, Prevention, Council for High Blood Pressure R, Council on Peripheral Vascular D, Interdisciplinary Council on Quality of $\mathrm{C}$ and Outcomes R: Guidelines for the primary prevention of stroke: a guideline for healthcare professionals from the American Heart Association/American Stroke Association. Stroke, 2011; 42: $517-584$

10) Graham I, Atar D, Borch-Johnsen K, Boysen G, Burell G, Cifkova R, Dallongeville J, De Backer G, Ebrahim S, Gjelsvik B, Herrmann-Lingen C, Hoes A, Humphries S, Knapton M, Perk J, Priori SG, Pyorala K, Reiner Z, Ruilope L, Sans-Menendez S, Scholte op Reimer W,
Weissberg P, Wood D, Yarnell J, Zamorano JL, Walma E, Fitzgerald T, Cooney MT, Dudina A and European Society of Cardiology Committee for Practice G: European guidelines on cardiovascular disease prevention in clinical practice: executive summary: Fourth Joint Task Force of the European Society of Cardiology and Other Societies on Cardiovascular Disease Prevention in Clinical Practice (Constituted by representatives of nine societies and by invited experts). Eur Heart J, 2007; 28: 2375-2414

11) Castelli WP: The new pathophysiology of coronary artery disease. Am J Cardiol, 1998; 82: 60T-65T

12) Storkus WJ, Salter RD, Cresswell P and Dawson JR: Peptide-induced modulation of target cell sensitivity to natural killing. J Immunol, 1992; 149: 1185-1190

13) Rizzo M, Pernice V, Frasheri A, Di Lorenzo G, Rini GB, Spinas GA and Berneis K: Small, dense low-density lipoproteins (LDL) are predictors of cardio- and cerebro-vascular events in subjects with the metabolic syndrome. Clin Endocrinol (Oxf), 2009; 70: 870-875

14) National Cholesterol Education Program Expert Panel on Detection E and Treatment of High Blood Cholesterol in A: Third Report of the National Cholesterol Education Program (NCEP) Expert Panel on Detection, Evaluation, and Treatment of High Blood Cholesterol in Adults (Adult Treatment Panel III) final report. Circulation, 2002; 106: 3143-3421

15) Brott T, Adams HP, Jr., Olinger CP, Marler JR, Barsan WG, Biller J, Spilker J, Holleran R, Eberle R, Hertzberg $\mathrm{V}$ and et al.: Measurements of acute cerebral infarction: a clinical examination scale. Stroke, 1989; 20: 864-870

16) Sulter G, Steen C and De Keyser J: Use of the Barthel index and modified Rankin scale in acute stroke trials. Stroke, 1999; 30: 1538-1541

17) Meyer BC, Hemmen TM, Jackson CM and Lyden PD: Modified National Institutes of Health Stroke Scale for use in stroke clinical trials: prospective reliability and validity. Stroke, 2002; 33: 1261-1266

18) Quinn TJ, McArthur K, Dawson J, Walters MR and Lees KR: Reliability of structured modified rankin scale assessment. Stroke, 2010; 41: e602; author reply e603

19) Adams HP, Jr., Bendixen BH, Kappelle LJ, Biller J, Love BB, Gordon DL and Marsh EE, 3rd: Classification of subtype of acute ischemic stroke. Definitions for use in a multicenter clinical trial. TOAST. Trial of Org 10172 in Acute Stroke Treatment. Stroke, 1993; 24: 35-41

20) Banks JL and Marotta CA: Outcomes validity and reliability of the modified Rankin scale: implications for stroke clinical trials: a literature review and synthesis. Stroke, 2007; 38: 1091-1096

21) Kalogirou M, Tsimihodimos V, Gazi I, Filippatos T, Saougos V, Tselepis AD, Mikhailidis DP and Elisaf M: Effect of ezetimibe monotherapy on the concentration of lipoprotein subfractions in patients with primary dyslipidaemia. Current medical research and opinion, 2007; 23: 1169-1176

22) Roques F, Michel P, Goldstone AR and Nashef SA: The logistic EuroSCORE. Eur Heart J, 2003; 24: 881-882

23) QiaoZhen X, AiGuo M, Tong W, JingJing L and HaiYing L: Correlation between of small dense low-density lipoprotein cholesterol with acute cerebral infarction and carotid atherosclerotic plaque stability. Journal of clinical 
laboratory analysis, 2019; 33: e22891

24) Shen H, Zhou J, Shen G, Yang H, Lu Z and Wang H: Correlation between serum levels of small, dense lowdensity lipoprotein cholesterol and carotid stenosis in cerebral infarction patients $>65$ years of age. Annals of vascular surgery, 2014; 28: 375-380

25) Zeljkovic A, Vekic J, Spasojevic-Kalimanovska V, JelicIvanovic Z, Bogavac-Stanojevic N, Gulan B and Spasic S: LDL and HDL subclasses in acute ischemic stroke: prediction of risk and short-term mortality. Atherosclerosis, 2010; 210: 548-554

26) Zhao Y, Yao Z, D’Souza W, Zhu C, Chun H, Zhuoga C, Zhang Q, Hu X and Zhou D: An epidemiological survey of stroke in Lhasa, Tibet, China. Stroke, 2010; 41: 27392743

27) Grundy SM, Cleeman JI, Merz CN, Brewer HB, Jr., Clark LT, Hunninghake DB, Pasternak RC, Smith SC, Jr., Stone NJ and Coordinating Committee of the National Cholesterol Education P: Implications of recent clinical trials for the National Cholesterol Education Program Adult Treatment Panel III Guidelines. J Am Coll Cardiol, 2004; 44: 720-732

28) Farwell WR, Sesso HD, Buring JE and Gaziano JM: Nonhigh-density lipoprotein cholesterol versus low-density lipoprotein cholesterol as a risk factor for a first nonfatal myocardial infarction. Am J Cardiol, 2005; 96: 11291134

29) Liu J, Sempos CT, Donahue RP, Dorn J, Trevisan M and Grundy SM: Non-high-density lipoprotein and very-lowdensity lipoprotein cholesterol and their risk predictive values in coronary heart disease. Am J Cardiol, 2006; 98: 1363-1368

30) Albers JJ, Kennedy H and Marcovina SM: Evaluation of a new homogenous method for detection of small dense LDL cholesterol: comparison with the LDL cholesterol profile obtained by density gradient ultracentrifugation. Clin Chim Acta, 2011; 412: 556-561
31) Oravec S, Dukat A, Gavornik P, Caprdna M and Kucera M: [Serum lipoprotein profile in newly recognized arterial hypertension. The role of atherogenic lipoproteins in the pathogenesis of disease]. Vnitr Lek, 2010; 56: 967-971

32) Packard CJ: Triacylglycerol-rich lipoproteins and the generation of small, dense low-density lipoprotein. Biochem Soc Trans, 2003; 31: 1066-1069

33) Gardner CD, Fortmann SP and Krauss RM: Association of small low-density lipoprotein particles with the incidence of coronary artery disease in men and women. JAMA, 1996; 276: 875-881

34) Stampfer MJ, Krauss RM, Ma J, Blanche PJ, Holl LG, Sacks FM and Hennekens CH: A prospective study of triglyceride level, low-density lipoprotein particle diameter, and risk of myocardial infarction. JAMA, 1996; 276: 882888

35) Kayran Y, Yayla V, Cabalar M, Bajrami A, Karamanli Y, Gedikbasi A and Tanriverd IZ: LDL Subclasses in Ischemic Stroke: A Risk Factor? Noro Psikiyatr Ars, 2019; 56: $13-17$

36) Xu T, Zhang JT, Yang M, Zhang H, Liu WQ, Kong Y, Xu $\mathrm{T}$ and Zhang YH: Dyslipidemia and outcome in patients with acute ischemic stroke. Biomed Environ Sci, 2014; 27: 106-110

37) Xing Y, An Z, Yu N, Zhao W, Ning X and Wang J: Low Density Lipoprotein Cholesterol and the Outcome of Acute Ischemic Stroke: Results of a Large Hospital-Based Study. Eur Neurol, 2016; 76: 195-201

38) Li W, Liu M, Wu B, Liu H, Wang LC and Tan S: Serum lipid levels and 3-month prognosis in Chinese patients with acute stroke. Adv Ther, 2008; 25: 329-341

39) Koren-Morag N, Tanne D, Graff E and Goldbourt U: Low- and high-density lipoprotein cholesterol and ischemic cerebrovascular disease: the bezafibrate infarction prevention registry. Arch Intern Med, 2002; 162: 993999 


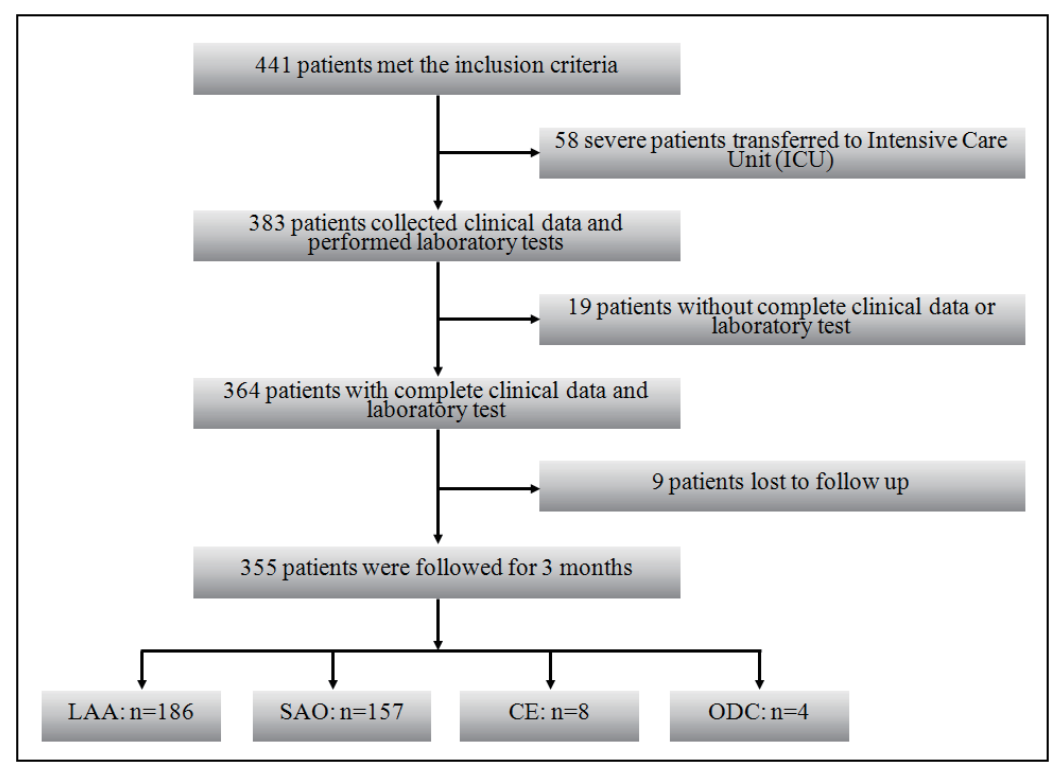

Supplementary Fig. 1. The flow chart of patient selection

Supplementary Table 1. Correlation analysis between sdLDL-C and other variables in AIS patients

\begin{tabular}{lcc}
\hline Variable & $r$ & $P$ value \\
\hline Age & 0.068 & 0.198 \\
Hypertension & 0.013 & 0.809 \\
Diabetes & 0.057 & 0.282 \\
Glucose & 0.149 & 0.005 \\
TC & 0.454 & 0.000 \\
TG & 0.163 & 0.002 \\
LDL-C & 0.545 & 0.000 \\
HDL-C & -0.033 & 0.535 \\
\hline
\end{tabular}

Supplementary Table 2. Comparison of clinical characteristics with AIS subtypes

\begin{tabular}{|c|c|c|c|c|}
\hline Variables & LAA $(n=186)$ & SAO $(n=157)$ & $\mathrm{CE}(n=8)$ & $\mathrm{ODC}(n=4)$ \\
\hline Male / Female & $119(63.98) / 67(36.02)^{\mathrm{a}}$ & $86(54.78) / 71(45.22)^{a}$ & $7(87.50) / 1(12.50)^{a}$ & $2(50.00) / 2(50.00)^{a}$ \\
\hline NIHSS* & $6.62 \pm 6.88^{\mathrm{ac}}$ & $5.31 \pm 6.03^{b c}$ & $10.12 \pm 6.15^{\mathrm{a}}$ & $2.50 \pm 2.06^{\mathrm{c}}$ \\
\hline $\mathrm{mRS}^{*}$ & $1.17 \pm 1.47^{\mathrm{a}}$ & $0.85 \pm 1.22^{b}$ & $2.12 \pm 1.54^{\mathrm{c}}$ & $0.75 \pm 0.83^{\mathrm{abc}}$ \\
\hline Glucose $(\mathrm{mmol} / \mathrm{L})^{*}$ & $6.95 \pm 2.45^{\mathrm{a}}$ & $6.47 \pm 2.16^{\mathrm{a}}$ & $6.31 \pm 1.22^{\mathrm{ab}}$ & $5.83 \pm 0.69^{b}$ \\
\hline Diabetes & $60(32.26)^{\mathrm{a}}$ & $33(21.02)^{b}$ & $1(12.50)^{a b}$ & $1(25.00)^{a b}$ \\
\hline $\mathrm{TC}(\mathrm{mmol} / \mathrm{L})$ & $3.85 \pm 0.97^{\mathrm{a}}$ & $3.83 \pm 0.85^{\mathrm{a}}$ & $3.45 \pm 0.55^{\mathrm{a}}$ & $3.61 \pm 0.77^{\mathrm{a}}$ \\
\hline $\mathrm{TG}(\mathrm{mmol} / \mathrm{L})$ & $1.46 \pm 0.83^{\mathrm{a}}$ & $1.40 \pm 1.36^{b}$ & $0.99 \pm 0.22^{b}$ & $1.03 \pm 0.27^{\mathrm{ab}}$ \\
\hline LDL-C (mmol/L) & $2.19 \pm 0.66^{\mathrm{a}}$ & $2.18 \pm 0.65^{\mathrm{a}}$ & $2.04 \pm 0.52^{\mathrm{a}}$ & $1.83 \pm 0.61^{\mathrm{a}}$ \\
\hline LDL1-C (mg/dl) & $17.73 \pm 7.53^{\mathrm{a}}$ & $19.22 \pm 8.72^{\mathrm{a}}$ & $17.38 \pm 5.34^{\mathrm{a}}$ & $17.25 \pm 3.56^{\mathrm{a}}$ \\
\hline LDL2-C (mg/dl) & $21.09 \pm 7.07^{\mathrm{a}}$ & $20.95 \pm 6.67^{\mathrm{a}}$ & $23.88 \pm 4.75^{\mathrm{a}}$ & $21.00 \pm 4.06^{\mathrm{a}}$ \\
\hline Drinker & $52(27.96)^{\mathrm{a}}$ & $40(25.47)^{\mathrm{a}}$ & $0(0)^{a}$ & $0(0)^{\mathrm{a}}$ \\
\hline Statin therapy & $7(3.76)^{a}$ & $4(2.55)^{a}$ & $1(12.50)^{\mathrm{a}}$ & $1(25.00)^{a}$ \\
\hline
\end{tabular}

Categorical variables presented as numbers and proportions (\%), continuous variables as mean \pm standard deviation (SD) *

Groups that are not labeled with any of the same letters (a, b, c) indicate statistically significant at 0.05 .

Mann whitney $U$ test for continuous values and chi-square test for discrete values 
Supplementary Table 3. Correlation between clinical characteristics and stroke outcomes

\begin{tabular}{|c|c|c|c|c|}
\hline Variables & Mortality $(n=4)$ & Dependency $(n=47)$ & Independency $(n=304)$ & $P$ value \\
\hline Age (year) ${ }^{*}$ & $54.50 \pm 11.67$ & $65.49 \pm 9.59$ & $64.20 \pm 10.82$ & 0.261 \\
\hline Male / Female & $0(0.00) / 4(100.00)$ & $21(44.68) / 26(55.32)$ & $193(63.49) / 111(36.51)$ & 0.002 \\
\hline $\operatorname{BMI}\left(\mathrm{kg} / \mathrm{m}^{2}\right)^{*}$ & $21.20 \pm 1.87$ & $23.14 \pm 2.54$ & $23.61 \pm 2.81$ & 0.135 \\
\hline \multicolumn{5}{|l|}{ TOAST } \\
\hline LAA & $2(50.00)$ & $29(61.70)$ & $155(50.99)$ & 0.391 \\
\hline SAO & $1(25.00)$ & $17(36.17)$ & $139(45.72)$ & 0.349 \\
\hline $\mathrm{CE}$ & $0(0)$ & $1(2.12)$ & $7(2.30)$ & 0.952 \\
\hline ODC & $1(25.00)$ & $0(0.00)$ & $3(0.99)$ & 0.000 \\
\hline UC & 0 & 0 & 0 & \\
\hline NIHSS* & $15.00 \pm 6.56$ & $16.06 \pm 7.66$ & $4.41 \pm 4.58$ & 0.000 \\
\hline $\mathrm{mRS}^{*}$ & $2.00 \pm 1.86$ & $2.64 \pm 1.39$ & $0.78 \pm 1.18$ & 0.000 \\
\hline Glucose $(\mathrm{mmol} / \mathrm{L})^{*}$ & $6.71 \pm 1.96$ & $7.39 \pm 2.58$ & $6.60 \pm 2.24$ & 0.003 \\
\hline Hypertension & $4(100.00)$ & $33(70.21)$ & $219(72.04)$ & 0.443 \\
\hline Diabetes & $3(75.00)$ & $14(29.78)$ & $78(25.66)$ & 0.076 \\
\hline Smoker & $0(0.00)$ & $11(23.40)$ & $96(31.58)$ & 0.291 \\
\hline Drinker & $0(0.00)$ & $10(21.28)$ & 82 (26.97) & 0.349 \\
\hline Statin therapy & $2(50.00)$ & $1(2.12)$ & $10(3.29)$ & 0.000 \\
\hline
\end{tabular}

Categorical variables presented as numbers and proportions (\%), continuous variables as mean \pm standard deviation $(\mathrm{SD}) *$

The Kruskal-Wallis $\mathrm{H}$ test for continuous values and chi-square test for discrete values

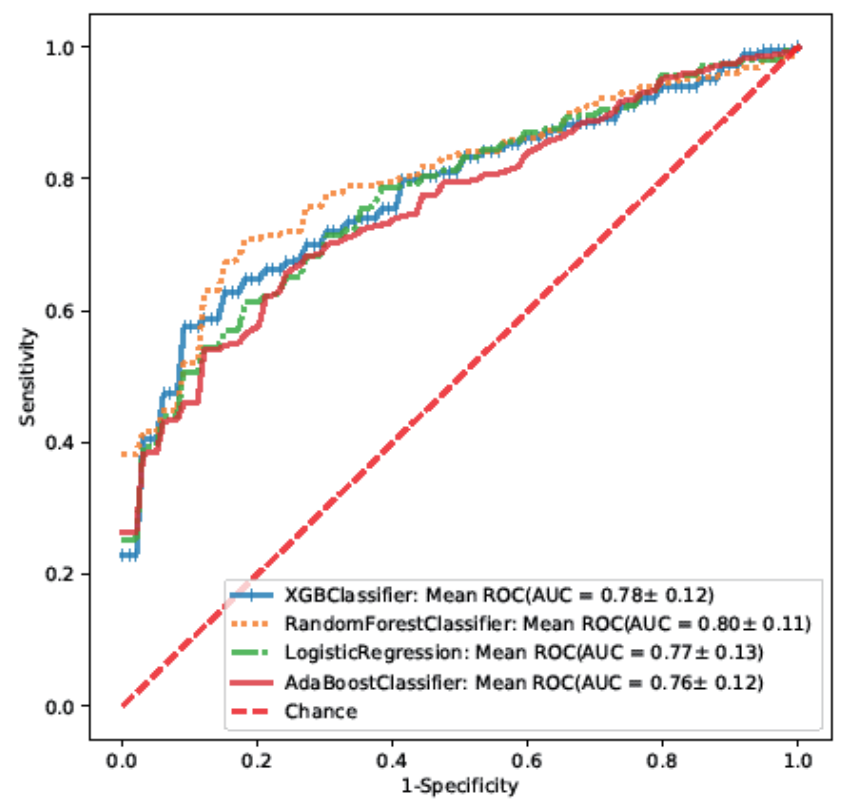

Supplementary Fig. 2. Receiver operating characteristic (ROC) curve and area under the curve (AUC) for multiple classifiers including XGBoost, Random Forest, Logistic Regression, and AdaBoost 
A
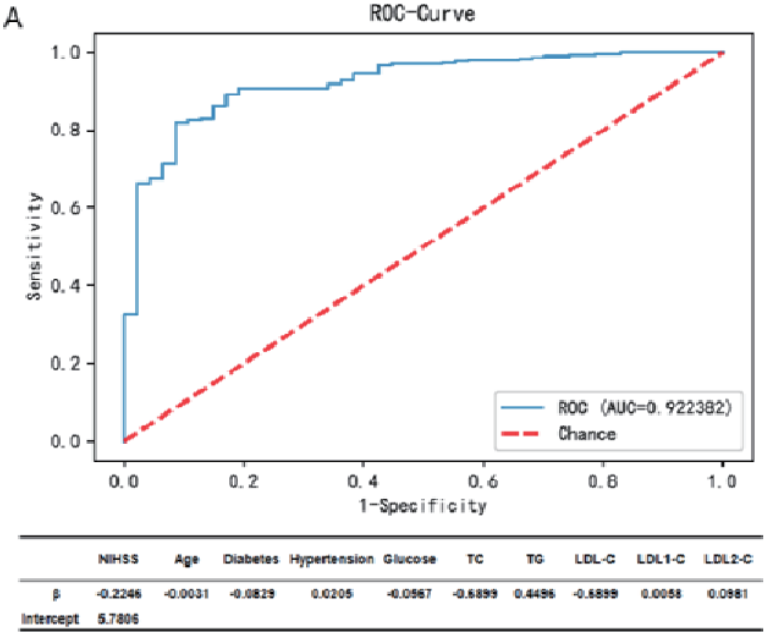

C
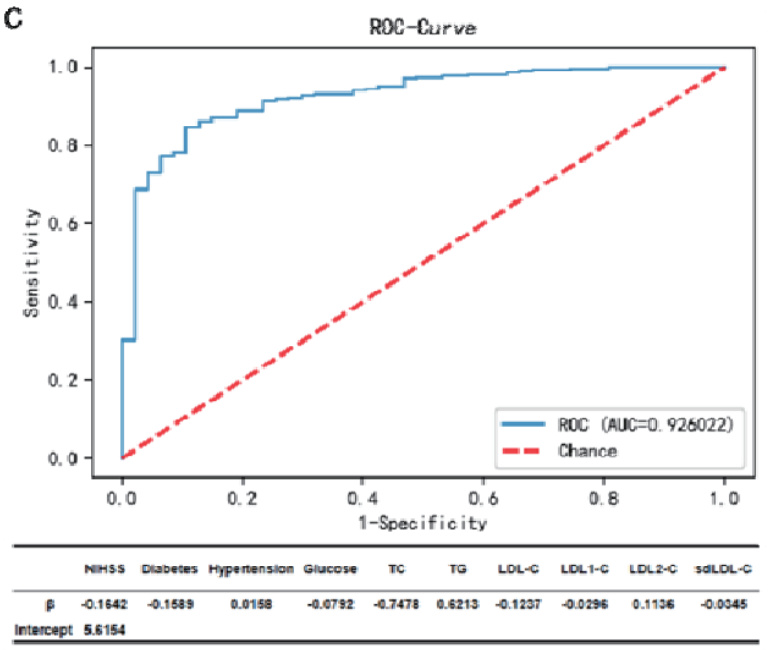
Intercept 5.6154

E

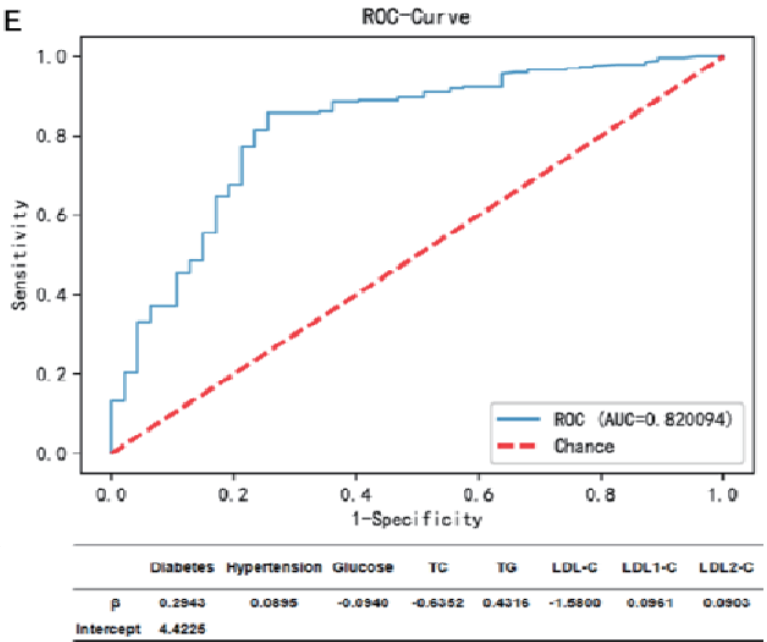

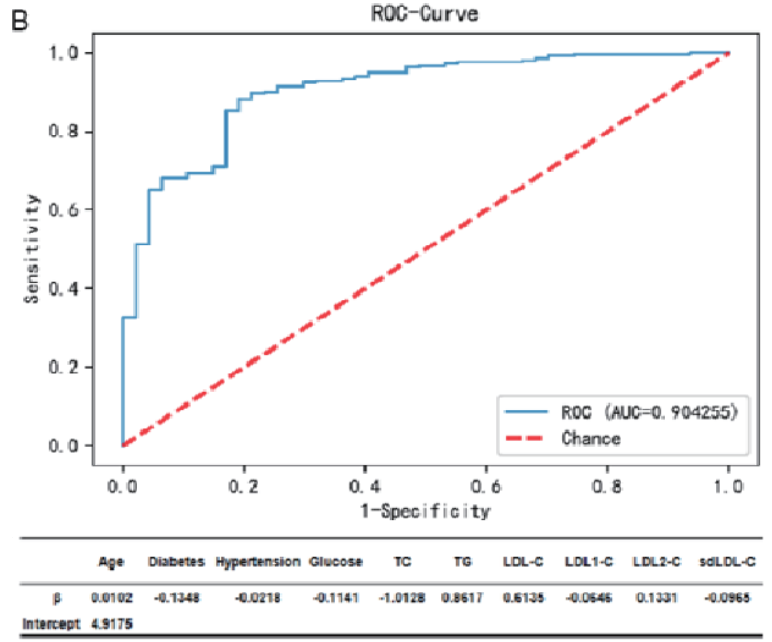

D

D ROC-Curve

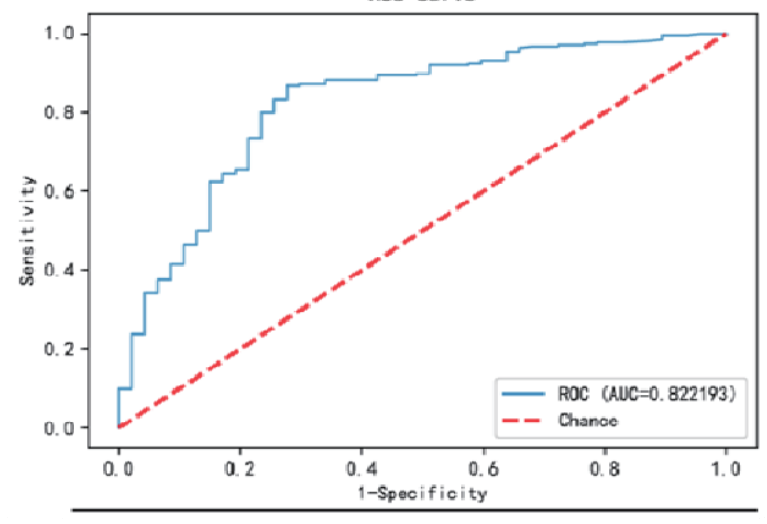

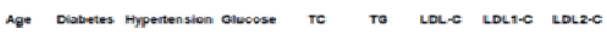

\begin{tabular}{llllllllll}
\hline B & 0.0111 & 0.0064 & 0.1418 & -0.0910 & -0.6255 & 0.3984 & -1.5945 & 0.0944 & 0.0923
\end{tabular} Intercept 5.1147

Supplementary Fig. 3. Logistic regression models based on different variables

(A) A model of prognosis prediction without sdLDL-C variable. (B) A model of prognosis prediction without NIHSS variable. (C) A model of prognosis prediction without age variable. (D) A model of prognosis prediction without sdLDL-C and NIHSS. (E) A model of prognosis prediction without sdLDL-C, NIHSS and age. 
Supplementary Table 4. The intercept and slope of two logical regression models

\begin{tabular}{|c|c|c|c|c|c|c|c|}
\hline \multicolumn{4}{|c|}{ A model for AIS risk } & \multicolumn{4}{|c|}{ A model for AIS prognosis } \\
\hline Diabetes & -0.1143 & $-0.745,0.516$ & 0.8828 & Age & 0.0047 & $-0.045,0.054$ & 5.3350 \\
\hline Hypertension & 0.5305 & $0.083,0.978$ & & Diabetes & -0.1673 & $-1.247,0.912$ & \\
\hline Glucose & 0.1093 & $-0.037,0.256$ & & Hypertension & -0.0065 & $-1.008,0.995$ & \\
\hline TC & 0.6468 & $0.087,1.206$ & & NIHSS & -0.1631 & $-0.264,-0.062$ & \\
\hline LDL-C & -0.2140 & $-0.935,0.507$ & & $\mathrm{TC}$ & -0.7566 & $-1.821,0.308$ & \\
\hline LDL1-C & -0.1092 & $-0.144,-0.074$ & & TG & 0.6421 & $-0.171,1.455$ & \\
\hline LDL2-C & -0.0429 & $-0.081,-0.005$ & & LDL-C & -0.1089 & $-1.665,1.447$ & \\
\hline \multirow[t]{3}{*}{ sdLDL-C } & 0.0381 & $0.012,0.064$ & & LDL1-C & -0.0298 & $-0.111,0.051$ & \\
\hline & & & & LDL2-C & 0.1134 & $0.026,0.201$ & \\
\hline & & & & sdLDL-C & -0.0353 & $-0.080,0.010$ & \\
\hline
\end{tabular}

